\title{
A planar respiration sensor based on a capaciflector structure
}

\author{
Neil M. White ${ }^{11^{*}}$, Jordan Ash ${ }^{1}$, Yang Wei ${ }^{1}$ and Harry Akerman ${ }^{2}$ \\ ${ }^{1}$ Department of Electronics and Computer Science, University of Southampton, SO17 1BJ, United Kingdom \\ ${ }^{2}$ University Hospital Southampton NHS Foundation Trust, Tremona Road, Southampton, SO16 6YD, United Kingdom \\ * Senior Member, IEEE
}

\begin{abstract}
Received XX XXX 2017, revised XX XXX 2017, accepted XX XXX 2017, published XX XXX 2016, current version XX XXX 2017. (Dates will be inserted by IEEE; "published" is the date the accepted preprint is posted on IEEE Xplore ${ }^{\circledR}$; "current version" is the date the typeset version is posted on $X$ plore ${ }^{\circledR}$ ).
\end{abstract}

\begin{abstract}
Respiratory diseases such as asthma and chronic obstructive pulmonary disease (COPD) affect more than 300 million people worldwide. Devices such as the pneumotachograph are currently used within a clinical environment for measuring inhalation, expiration and respiration cycle but are physically large and not suitable for home use by patients. A small, lightweight respiration sensor for use in the home environment, allows patients to monitor respiratory rate in a simple manner. The capaciflector was originally developed by NASA as a capacitive proximity sensor for collision detection in robots. We have found that the device can also be used to detect breathing patterns in humans by attaching it to the chest. In this letter, we discuss the simulation, construction and testing of a capaciflector for use as a respiration sensor and describe how it can be interfaced to a microcontroller in order to allow wireless data transmission over a Wi-Fi signal.
\end{abstract}

Index Terms- Medical sensor, capaciflector, planar respiration sensor, sensor interfacing

\section{INTRODUCTION}

Home healthcare devices and activity trackers such as Fitbit [1] are now widely used by a variety of people to monitor their physical activities such as heart rate and step counting, but respiration rate is not currently measured. For those with respiratory diseases such as asthma and COPD, this parameter is of great significance and a variety of benefits can be envisaged if patients are able to monitor this in their home environment, particularly in scenarios where they are becoming septic and the breathing pattern is affected. Clinical respiratory sensing systems such as the pneumotachograph and inductive plethysmograph are not particularly amenable for home use as they are designed for specialist, clinical use. A simple and potentially low-cost respiration sensor that can be coupled with an existing infrastructure and smartphone App, would therefore appear to be desirable.

The capaciflector takes its name from capacitive reflector and was developed by the NASA Goddard Space Flight Center [2] in the early 1990s as a proximity sensor for collision detection in robots. We have found that by attaching such devices to the chest, it is possible to obtain a stable signal that allows respiration rate to be detected. Capaciflectors are essentially planar devices and can therefore be attached to the human body in a similar manner to biomedical hydrogel electrodes. The devices are small, lightweight and can be made from inexpensive materials. They can be interfaced to a variety of commercial microcontroller systems in a relatively straightforward manner to allow them to be configured as wireless sensors (via Bluetooth, Wi-Fi, ZigBee etc.)

\section{THE CAPACIFLECTOR AS A RESPIRATION SENSOR}

The capaciflector is essentially a modified form of a planar capacitor structure. It has a third electrode between the ground plane and sensor electrode. This is termed the reflector electrode and its purpose is to project the electric flux out of plane in the direction of the object being detected. The structure of the capaciflector is shown in Fig. 1.

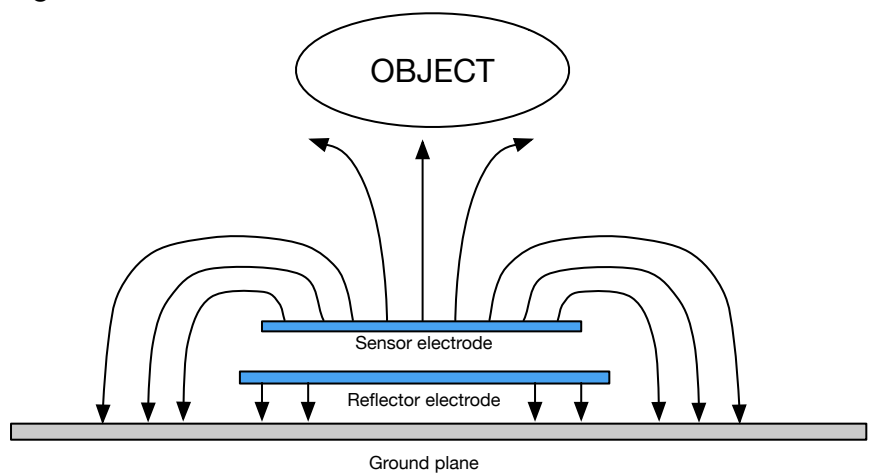

Fig. 1. The structure of a capaciflector depicting the three electrodes and direction of electric flux.

The sensor and reflector electrodes are driven at the same electric potential. As an object moves towards the capaciflector (or changes its permittivity in some manner) then the result is a change in capacitance, which can be measured. When used as a respiration sensor the device is mounted on the chest, which becomes the 'object' 
depicted in Fig 1. Use of the capaciflector in this configuration has not been previously described in the literature, but has been the subject of unpublished reports at the University of Southampton, UK [3] and the University of Virginia, USA [4].

\section{A. Capaciflector construction}

A prototype capaciflector was made using copper foil $(100 \mu \mathrm{m}$ thickness) for the electrode material and paper as the dielectric layers, which separates the electrodes. An additional paper layer was also used to insulate the sensor electrode from the human body. The multilayered device was mounted within a 3D printed housing, which was printed on an Objet Connex 350. This printer has the capability producing materials with varying degrees of compliance, from flexible through to rigid hence providing a range of mounting options to the chest (conformal or rigid). Fig. 2 shows an exploded view of the prototype device.

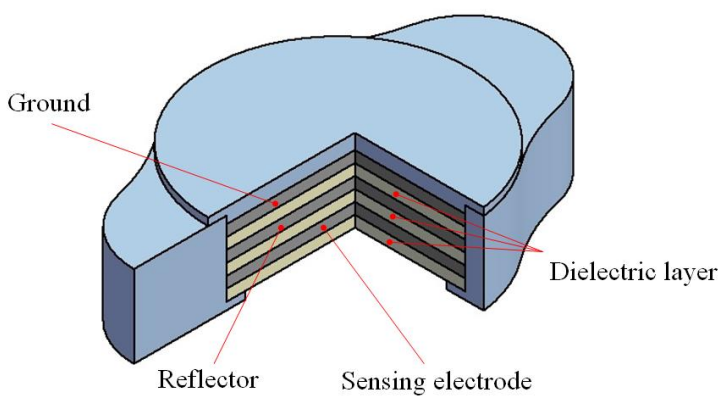

Fig. 2. An exploded view of the capaciflector showing the electrode and dielectric layers, together with the $3 \mathrm{D}$ printed housing (not to scale).

The concept of using the device as a respiration sensor requires the sensing electrode to be mounted close to the chest. During respiration, the chest cavity expands and the skin also stretches. Human skin comprises many different layers including the epidermis, dermis, subcutis and a variety of nerves, fibers and fat. The dielectric properties of the skin have been documented and described by Gabriel et al [5] and the complex features contributing to the permittivity will change as the skin stretches.

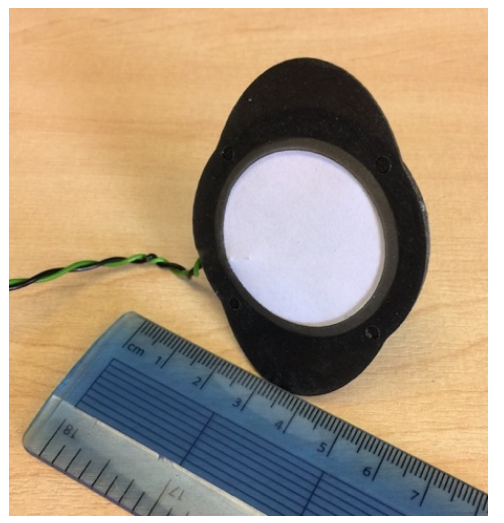

Fig. 3. A photograph of the actual device showing a scale and the 3D printed package.
Fig. 3 shows a photograph of the actual device. The diameter of the electrodes is $40 \mathrm{~mm}$ and, with the addition of the $3 \mathrm{D}$ printed packaging, this results in a device that is around $60 \mathrm{~mm}$ in length and $50 \mathrm{~mm}$ in width. The total thickness of the active layers, including the packaging is $4 \mathrm{~mm}$. A total of 4 different compliance settings were chosen on the $3 \mathrm{D}$ printer so that it was possible to assess the effect of the packaging stiffness on the device performance. The most repeatable results were obtained with the device that allowed a small amount of flexing, yet remained rigid when mounted on the chest.

\section{B. Capaciflector simulation}

The behavior of the capaciflector was simulated using the COMSOL Multiphysics ${ }^{\circledR}$ package. The copper electrodes and paper insulation layers were modelled in close proximity with an object. The surrounding medium was modelled as being air (relative permittivity 1) and the object was positioned $3 \mathrm{~mm}$ away from the sensing electrode. The object is a crude representation of the chest and was modelled as a $3 \mathrm{~cm}$ thick cylinder of diameter $15 \mathrm{~cm}$ having the same permittivity as water ( 80 at room temperature). This is a very approximate estimation, as the skin is a complex organ and its permittivity is function of frequency and the permittivity varies in the various layers at different depths [6]. The outer, thin layer (stratum corneum), of approximate thickness $20 \mu \mathrm{m}$, has a relative permittivity of around 1000. Water content increases with depth and hence this value decreases to about 80 . Nonetheless, this allows the electric field vectors to be observed. The results are shown in Fig. 4 and indicate that an object placed close to the device will cause a change in the electrical flux surrounding the sensor. The electric field vectors can be clearly seen to exist outside the parallel plate structure (as opposed to a simple planar capacitor) and towards the object, which they do not penetrate and are reflected back towards the capaciflector.

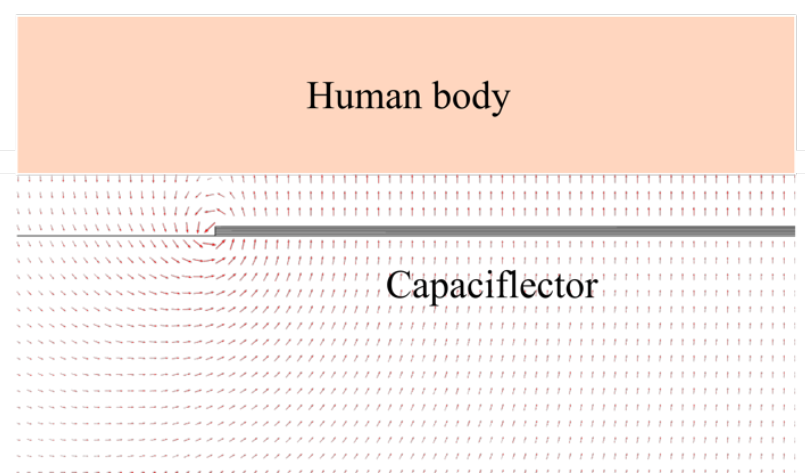

Fig. 4. A simulation of the capaciflector showing the effect of an object on the electrical flux surrounding the device.

Fig. 5 shows a graph depicting the electric field strength along a line through the central axis of the capaciflector up to a distance of $1 \mathrm{~cm}$ from the capaciflector. The plot has been truncated on the y-axis in order to show how the electric field (in air) varies outside the capaciflector volume because the electric field between the reflector electrode and ground is $5 \mathrm{~V}$ across a $0.1 \mathrm{~mm}$ gap $(50 \mathrm{kV} / \mathrm{m})$ and hence would dominate the plot. At the sense electrode, the field strength is $220 \mathrm{~V} / \mathrm{m}$, which remains constant until it reaches the object $(3 \mathrm{~mm}$ away), where it collapses to zero. 


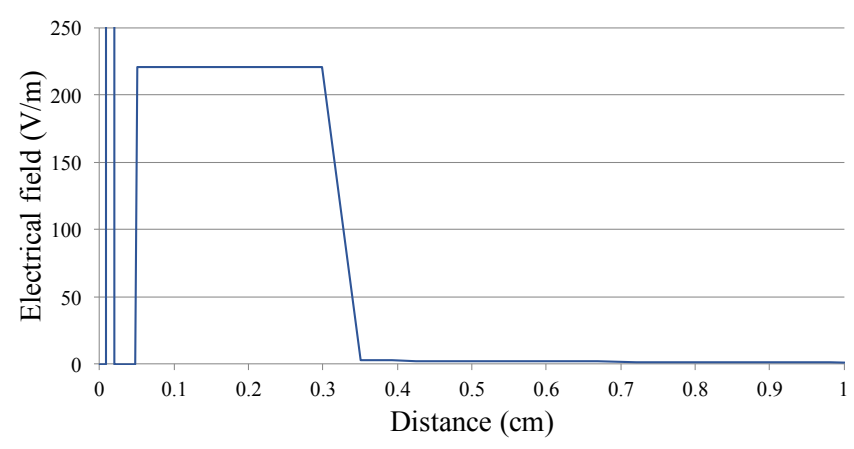

Fig. 5. A plot of electric field strength along the central axis outwards from the capaciflector. (Note that the plot has been truncated to remove the peak between the reflector and ground electrodes.)

\section{EXPERIMENTAL SETUP}

In order to investigate the suitability of using a capaciflector as a respiration sensor, an experiment was conducted so that the concept of a wireless system, which allows remote monitoring of the data, could be established. The capaciflector was configured as the active element in a relaxation oscillator. As the chest expands and contracts during the inhalation/exhalation cycle, the change in capacitance will result in a shift of frequency of the relaxation oscillator, which therefore produces a variable frequency square wave that can be fed directly into a general-purpose input/output pin (GIO) on a microcontroller, without the need for using an analog-to-digital converter.

Fig. 6 shows the system block diagram used for testing. The chosen single board computer was the low-cost Raspberry Pi 3, which has an inbuilt 802.11n Wireless LAN module. The generated data can therefore be monitored at any remote location with good WiFi reception. The capaciflector was mounted on the chest using doublesided medical-grade tape. Owing to the thickness of the packaging, the sensing electrode is at a distance of around $3 \mathrm{~mm}$ from the skin. A variety of suitable positions for mounting were investigated on the torso and also the back. A respiration signal was detected at all possible locations, but the most sensitive position is slightly left of center on the chest, in a region just below the pectoral muscle, as indicated in the diagram.

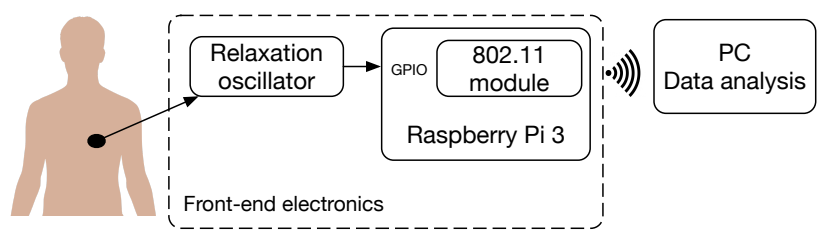

Fig. 6. A system block diagram of the test setup showing the capaciflector mounted on the chest, the front-end electronics and the remote data analysis capability provided by a WiFi connection.

Software was developed to run on the Raspberry Pi, which calculated the frequency of the waveform on the GPIO pin. This was achieved through the use of the WiringPi GPIO access library, which includes functions such as readDigitalPin, writeDigitalPin and
pinMode. The remote data analysis was accomplished using MATLAB, which contains an add-on support package for the Raspberry Pi. Communication between the two systems is effectively achieved through a single line of code in MATLAB, which gets the data through a WiFi connection.

The measured capacitance of the sensor between the ground and reflector electrodes was $100 \mathrm{pF}$ and this was used to set the freerunning frequency of the relaxation oscillator to be $100 \mathrm{kHz}$. The opamp used for the oscillator was a LT1259, which has a bandwidth of $90 \mathrm{MHz}$ and a slew rate of $1600 \mathrm{~V} / \mu$ s thereby allowing the oscillator to run at much higher frequencies, if desired. It was powered from a regulated $5 \mathrm{~V}$ source, which was also used to energize the sensing and reflector electrodes.

\section{EXPERIMENTAL RESULTS}

The sensor was mounted on the chest of a test subject and the frequency change of the oscillator and the time data were transmitted from the Raspberry Pi to a remote PC. The sampling rate was set to $20 \mathrm{~Hz}$ for all measurements. The volunteer was asked to sit in chair in a relaxed state and to take a series of breaths over a period of about 3 minutes and also to sit still and to breathe at a rate that they felt was 'normal'. Fig. 7 shows a typical time response plot of the sensor where the volunteer has attempted to breathe at a consistent rate over a period of about 220 seconds. During a full respiration cycle, the maximum frequency shift observed was found to be about $8 \mathrm{kHz}$.

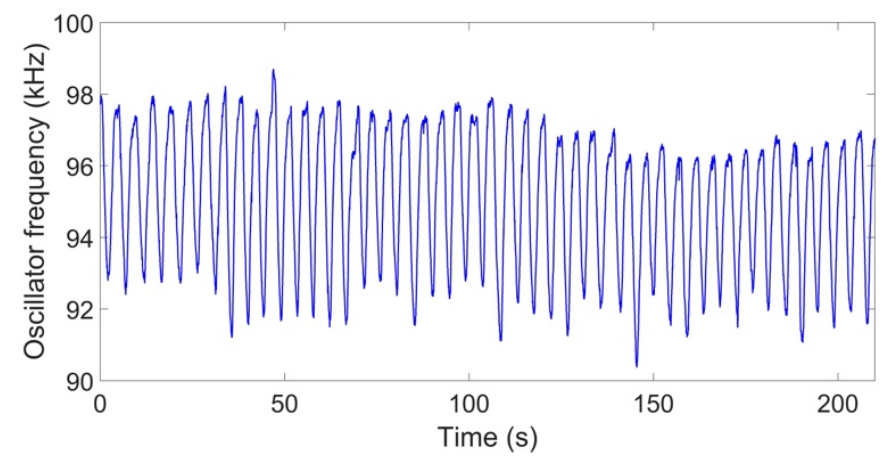

Fig. 7. The output of the oscillator (proportional to capacitance change) against time for regular breathing.

Fig. 8 shows a plot of the spectral response of the previous graph, obtained by taking a Fast Fourier Transform (FFT) of the time domain signal. This shows a dominant peak at a frequency of around $0.22 \mathrm{~Hz}$, equivalent to a breathing cycle of 4.6 seconds. The average range for an adult is typically between 12 to 18 breaths per minute, so the measured value is consistent with these figures. There are two other peaks with smaller amplitudes at around $0 \mathrm{~Hz}$ and $0.45 \mathrm{~Hz}$. The lower peak is likely to be due to drift in the capaciflector as there is no compensation for temperature in its current form. The higher frequency is due to the subject's slight body movement during the test. Use of a bandpass filter between 0.1 and $0.4 \mathrm{~Hz}$ would reject these frequencies and still allow observation of typical breathing rates. The series of experiments were conducted several times in order to create a meaningful data set, including on 6 different people. All subjects 
were generally healthy and in the age range 20-25.

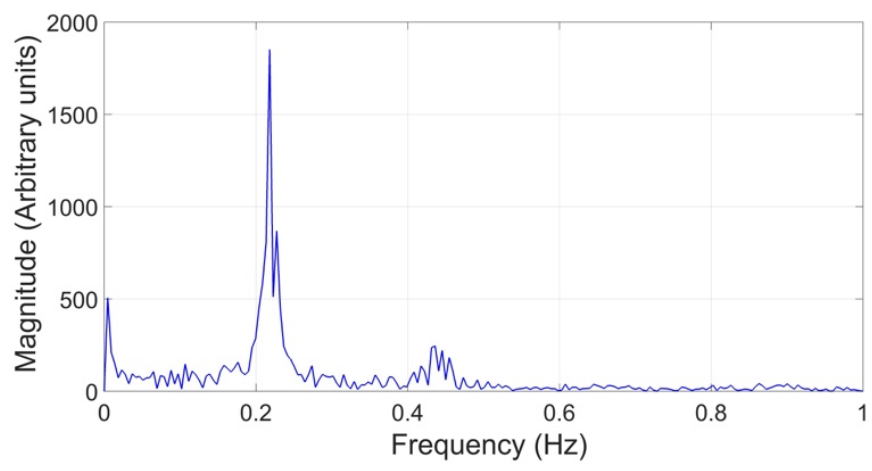

Fig. 8. A spectral plot of the time domain series in Fig. 6 showing a dominant peak at $0.22 \mathrm{~Hz}$.

\section{DISCUSSION}

The results show that it is possible to use a capaciflector to measure the respiration rate in humans. The exact mechanism by which this occurs requires further investigation. We have examined the possibility that bending of the sensor causes a change in capacitance. Flexing the structure does indeed result in a change, but when the structure was fixed into a rigid 3D printed housing to eliminate bend, it was still possible to get a signal corresponding to breathing rate. Therefore, the effect being measured is not simply due to a 'strain gauge' effect. Given that the capaciflector was initially developed to measure changes in electrical flux, it is highly likely that the change in permittivity of the skin as it stretches is a key factor. It is evident from the results that small changes in body position, twisting of the torso, mounting of the sensor etc. give rise to other discernable frequencies. These, however, are much smaller in magnitude than that of the respiration signal and can be removed by using a bandpass filter.

There is scope for developing a much more complex model in order to investigate further the behavior of the capaciflector/skin interaction. A more realistic model of the skin, with its many layers will help in this respect. Likewise, the choice of materials used in the capaciflector will make a significant difference to the overall performance and to the ease of construction.

Further tests were undertaken to examine how the capaciflector compared to two commercial systems, namely the pneumotachograph which requires the subject to wear a mask and chest-band inductance plethysmograph system. The pneumotachograph is generally accepted as the gold standard for respiratory measurement. The capaciflector and the plethysmograph gave an error of $+25 \%$ in respiration rate when compared to the measurement from the pneumotachograph. These devices are, however, based on three different measurement methods so the comparison is somewhat arbitrary.

There is clearly scope for improving the performance of the sensor. The device in its current form has no temperature compensation, for example, and this will clearly play a major role as a cross-sensitivity mechanism. Further work will aim to address this by the use of two capaciflectors operating as differential pair in order to reject the common-mode signals due to temperature variations. We have also noted that the reverse side of the device (facing away from the chest) responds to the presence of objects, so a shielding mechanism will need to be introduced in order to remove this as a source of interference.

\section{CONCLUSION}

This letter has shown that a capaciflector, normally used for proximity measurement, can be used as a respiration sensor by mounting the device on a human torso. The change in capacitance is strongly correlated to the breathing cycle. The device is potentially inexpensive to produce and can be configured as a wireless sensor and interfaced to a remote wireless system, or an app on a smartphone. Further research is required to investigate optimum material combinations and geometries for the device and also to fully establish the mechanism by which the respiration process causes the capacitance to change in a deterministic manner.

\section{REFERENCES}

[1] FitBit, May 2017. [Online]. Available: https://www.fitbit.com/uk/home

[2] J. M. Vranish, R. L. McConnell and S. Mahaligham, "Capaciflector collision avoidance sensors for robots", Computers \& Electrical Engineering, vol. 17, no. 3, pp. 173-179,1991.

[3] A. Aghajani, M. Buda, O. Bonner and T. Heslington, "Novel Approaches to Monitoring Breathing Pattern". School of Electronics and Computer Science Faculty of Engineering, Science and Mathematics University of Southampton. Unpublished. 2010.

[4] M. Holford, "Passive Non-Invasive Heart Rate and Breathing Monitor". [Online] Available: https://miraholford.wordpress.com/previous-work/passive-non-invasiveheart-rate-and-breathing-monitor, 2013

[5] C. Gabriel, S. Gabriel and E. Corthcout, "The dielectric properties of biological tissues: I. Literature survey,” Phys. Med. Biol, Vol. 41, pp. 2231-2249, 1996.

[6] S. Huclova, D. Erni and J. Frohlich, "Modelling and validation of dielectric properties of human skin in the MHz region focusing on skin layer morphology and material composition," J. Phys. D:Appl. Phys. Vol. 45, pp. doi:10.1088/00223727/45/2/025301, 2012. 